\title{
DENSIDADE E COMPOSIÇÃO FLORÍSTICA DO BANCO DE SEMENTES DE UM TRECHO DE FLORESTA ESTACIONAL SEMIDECIDUAL NO CAMPUS DA UNIVERSIDADE FEDERAL DE VIÇOSA, VIÇOSA, MG ${ }^{1}$
}

\author{
Brena Karina Siqueira Franco ${ }^{2}$, Sebastião Venâncio Martins ${ }^{3}$, Patricia Carneiro Lobo Faria ${ }^{4}$ e Guido \\ Assunção Ribeiro ${ }^{3}$
}

\begin{abstract}
RESUMO - O objetivo deste trabalho foi caracterizar o banco de sementes de um trecho de Floresta Estacional Semidecidual Secundária, por meio da análise florística e da densidade de sementes. Denominado Mata da Praça de Esportes, o fragmento está situado no Campus da UFV, em Viçosa, MG, cuja área corresponde a 10,65 ha em regeneração há 75 anos. A área amostral foi subdividida em 40 parcelas de 3 x $5 \mathrm{~m}$, distribuídas em quatro transectos paralelos. Amostras compostas foram feitas em 20 dessas parcelas e posteriormente distribuídas dentro de 40 caixas de madeira, em viveiro. O montante foi dividido, submetendo-se cada metade a sombreamentos de $11,5 \%$ e $60 \%$. A germinação total e das espécies arbustivo-arbóreas foi comparada nas duas condições de sombreamento, utilizando-se o teste $t$ para amostras independentes. O banco de sementes foi constituído predominantemente por ervas invasoras, pertencentes, em sua maioria, às famílias Asteraceae (15 espécies), Solanaceae (4 espécies) e Poaceae (3 espécies). Foram quantificadas 5.194 plântulas, com predomínio das pioneiras arbustivo-arbóreas das famílias Melastomataceae e Cecropiaceae. A germinação das sementes conduzida sob sombra foi significativamente maior do que no ambiente de menor sombreamento (luz). Espécies zoocóricas foram mais abundantes, indicando a importância dos fragmentos florestais como fonte de recurso e abrigo para a fauna local. A alta densidade de indivíduos de espécies arbóreas e arbustivas pioneiras, como Cecropia hololeuca, as espécies de Melastomataceae, Asteraceae e Piperaceae, importantes para a regeneração da floresta, reflete a capacidade de resiliência da área estudada, na ocorrência de distúrbios.
\end{abstract}

Palavras-chave: Ecologia de sementes, Densidade de sementes e Germinação.

\section{DENSITY AND FLORISTIC COMPOSITION OF THE SEMIDECIDUOUS SEASONAL FOREST STRETCH SEED BANK, LOCATED IN THE CAMPUS OF VIÇOSA FEDERAL UNIVERSITY, MG, BRAZIL}

\begin{abstract}
The objective of this paper was to characterize the seeds bank of a stretch at the Secondary Semideciduous Seasonal Forest, through the analysis of floristic aspects as well as seed density. This fragment is located on the UFV campus, in Viçosa, MG and is known as Mata da Praça de Esportes (Sports Court Forest), comprising an area of 10.65 ha under regeneration for 75 years. The sample area was subdivided into 40 plots of $3 \times 5 \mathrm{~m}$, distributed in 4 parallel transects. Composite samples were taken from 20 of those plots and then distributed within 40 wooden boxes, set up as seedling nurseries. Then, that amount was divided, allocating each half to shading levels of $11.5 \%$ and $60 \%$. The total germination, as well as the one of the arbustive-arboreous species, was compared for the two conditions of shade by the test $\mathrm{t}$ for independent samples. The seed bank has been comprised mainly of invading herbs, mostly belonging to the Asteraceae, Solanaceae and Poaceae (15, 4 and 3 species, respectively) families. A total of 5,194 seedlings have been recorded, mostly being arbustive-arboreous pioneer species of the Melastomataceae and Cecropiaceae families. The
\end{abstract}

\footnotetext{
${ }^{1}$ Recebido em 29.02.2008 e aceito para publicação em 19.04.2012

${ }^{2}$ Escola de Ensino Superior São Francisco de Assis, ESFA, Brasil. E-mail: <brenafranco@yahoo.com.br>.

${ }^{3}$ Universidade Federal de Viçosa, UFV, Brasil. E-mail: <venancio@ufv.br> e < gribeiro@ufv.br>.

${ }^{4}$ Universidade Federal de Juiz de Fora, UFJF, Brasil.
} 
seed germination carried out under shade was significantly higher than under the environment with $11.5 \%$ of shade (light). Zoochoric species were more abundant, indicating the importance of the Forest fragments as a source of resources and shelter for the local fauna. The high densities of pioneer arboreous and bush species such as Cecropia hololeuca, the species of the Melastomataceae, Asteraceae and Piperaceae, which are important for forest regeneration, indicate a resilience capacity of the studied area to restoration after disturbances.

Keywords: Seed ecology, Seed density and Germination.

\section{INTRODUÇÃO}

Atualmente, os remanescentes da cobertura vegetal nativa em diversos estados brasileiros estão muito reduzidos, como consequência da ocupação de novas áreas, tanto em ambientes rurais quanto urbanos. Muitas vezes, esses remanescentes se encontram isolados, sofrendo, ainda, perturbações pelo fogo, pela pecuária e retirada seletiva de madeira (OLIVEIRA FILHO et al., 1994). A vegetação florestal da Zona da Mata de Minas Gerais, onde está inserido o Município de Viçosa, também sofreu esse processo de fragmentação, que resultou em inúmeros pequenos fragmentos (VALVERDE, 1959; MEIRA-NETO et al., 1997), ou seja, florestas secundárias em processo de regeneração natural.

As florestas secundárias resultam de complexa combinação de diferentes fatores de perturbação antrópica, gerando um mosaico de ecossistemas secundários, extremamente diversos (UHL, 1987). Considerando que essas florestas se tornaram muito comuns nas regiões tropicais (BROWN; LUGO, 1990), a realização de estudos sobre esses ecossistemas é bastante relevante para se conhecer a estrutura, a diversidade e seu funcionamento e, portanto, propor medidas de conservação e manejo dessas comunidades, assim como fornecer subsídios a práticas de restauração de áreas degradadas.

A regeneração florestal é definida como o processo pelo qual a floresta perturbada atinge características de floresta madura (KLEIN, 1980), por meio de processos sucessionais que ocorrem após distúrbios. São fatores comuns de perturbação o corte e queima de árvores e arbustos, com posterior utilização do solo para atividades agropastoris, abertura de clareiras naturais causadas pela queda de uma ou mais árvores do dossel, deslizamentos, atividades vulcânicas e ataque de insetos (UHL et al., 1981; KAUFFMAN, 1991; COCHRANE; SCHULZE, 1999; MARTINS; RIBEIRO, 2002).

Revista Árvore, Viçosa-MG, v.36, n.3, p.423-432, 2012
O banco de sementes é constituído tanto por sementes produzidas na área quanto por aquelas transportadas de outros locais (HARPER, 1977), sendo essas viáveis, em estado de dormência primária ou induzida, presentes na superfície ou no interior do solo de determinada área (FENNER, 1985). É componente de extrema importância na recuperação de vegetações abertas (HARPER, 1977; PUTZ, 1983; SWAINE; HALL, 1983; UHL et al., 1988; GARWOOD, 1989), participando de processos ecológicos, como restabelecimento de comunidades após distúrbios e manutenção da diversidade de espécies, entre outros (PUTZ, 1983; SWAINE, HALL, 1983; LAWTON; PUTZ, 1988; GARWOOD, 1989; MARTINS et al., 2008; MARTINS, 2009).

O objetivo deste estudo foi caracterizar o banco de sementes de um trecho de Floresta Estacional Semidecidual Secundária, através da análise de sua composição florística e densidade de sementes. Isso para analisar alguns aspectos ecológicos desse componente do processo de regeneração e, assim, fornecer subsídios para atividades de manejo e conservação de fragmentos florestais ou de restauração de áreas degradadas.

\section{MATERIAL E MÉTODOS}

\section{1. Área de estudo}

Este estudo foi realizado num trecho de um fragmento florestal de 10,65 ha, localizado no Campus da Universidade Federal de Viçosa, no Município de Viçosa (2045'S e 42 $51^{\prime} \mathrm{W}$ ), Minas Gerais (Figura 1). O clima da região é Cwb, de acordo com a classificação de Köppen, com chuvas mal distribuídas ao longo do ano, verão chuvoso e inverno seco. O total pluviométrico anual médio é de $1.221 \mathrm{~mm}$ e a temperatura média, de $19^{\circ} \mathrm{C}$ (VIANELLO, 1991). O relevo é acidentado, fazendo parte do chamado domínio do "Mar de Morros". No topo de morros predomina o Latossolo VermelhoAmarelo, nas encostas das elevações o Latossolo 

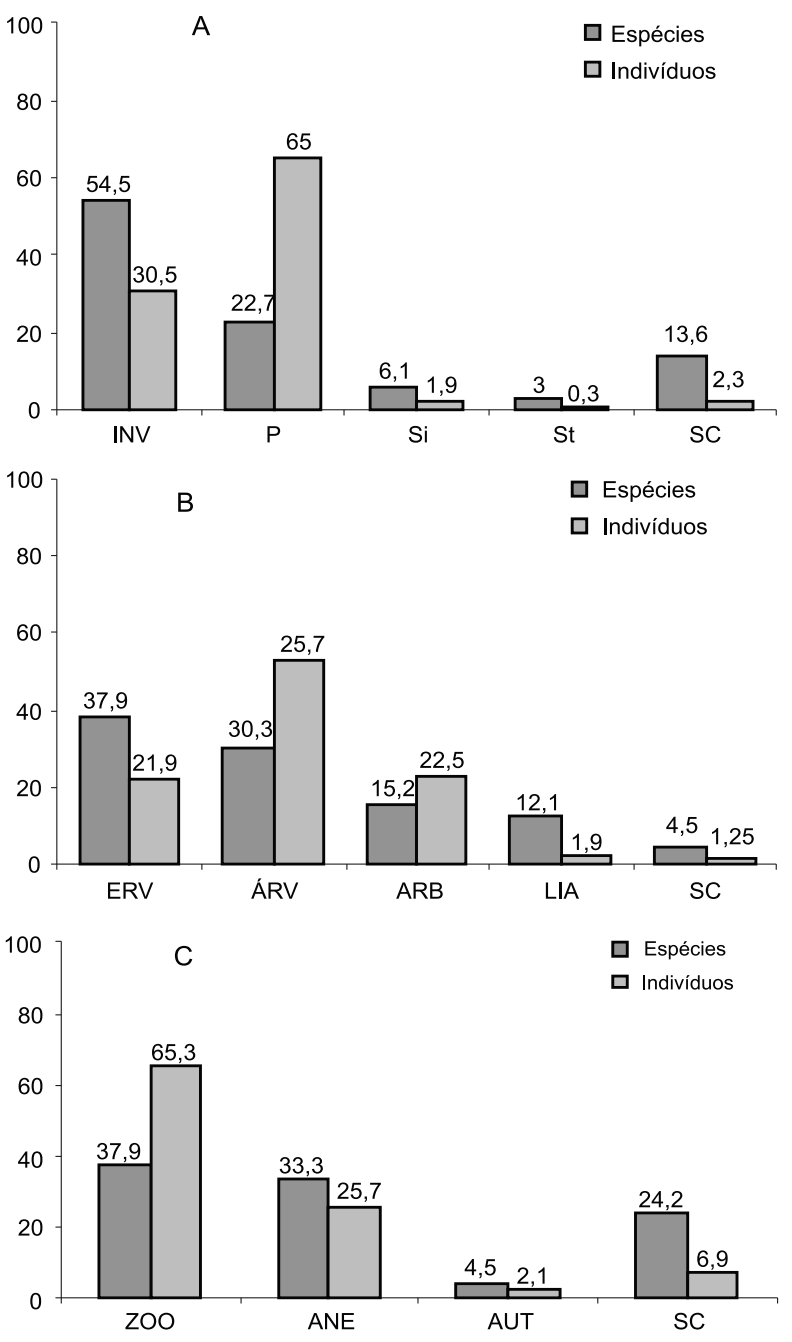

Figura 1 - Categorias sucessionais (a), formas de vida (b) e síndromes de dispersão (c) de todas as espécies amostradas no banco de sementes de um trecho da Mata da Praça de Esportes, Viçosa, MG, apresentadas em percentual de espécies e de indivíduos. ERV = Erva; ÁRV = Árvore; $\mathrm{ARB}=$ Arbusto; LIA = Liana; $\mathrm{SC}=$ Sem Caracterização; INV = Invasora; $\mathrm{P}=$ Pioneira $; \mathrm{Si}=$ Secundária inicial $; \mathrm{St}=$ Secundária tardia; $\mathrm{ZOO}=$ Zoocórica; ANE = Anemocórica; e AUT = Autocórica

Figure 1-Successional categories (a), life forms (b) and dispersal mode (c) of the all species sampled in the seed bank of the Mata da Praça de Esportes zone, Viçosa, MG, presented in percentage of individuals and species. $E R V=$ Herb $; A R V=$ Tree $; A R B=$ Shrub $; I A=$ Vine; $S C=$ Without Characterization; $I N V=$ Weed $P=$ Pioneer; $S i=$ Initial Secondary; $S t=$ Late Secondary; $Z O O=$ Zoochorous $;$ ANE = Anemochorous $;$ and $A U T=$ Autochorous.
Câmbico e no fundo dos vales o Neossolo Flúvico associado aos Gleissolos (REZENDE, 1971; CORRÊA, 1984; RESENDE, 1985; EMBRAPA, 1999). A vegetação natural da região é classificada como Floresta Estacional Semidecidual, de acordo com o Sistema Fisionômico Ecológico proposto por Veloso et al. (1991). De acordo com o histórico da região (GOLFARI, 1975), até a década de 1920 a área foi utilizada para cultivo do café (Coffea arabica). Dessa forma, pode-se atribuir a essa comunidade florestal cerca de 75 anos de idade.

\subsection{Banco de sementes}

Em um trecho visualmente conservado do fragmento e distante $100 \mathrm{~m}$ da borda, foram estabelecidos quatro transectos paralelos de $3 \times 50 \mathrm{~m}$, espaçados por $3 \mathrm{~m}$ e subdivididos em 10 parcelas de 3 x $5 \mathrm{~m}$. A coleta do banco de sementes foi realizada em abril de 2004, em 20 dessas parcelas, selecionadas de forma sistemática (todas de números pares), entre as quais foi feita uma amostragem composta, obtida em quatro pontos previamente sorteados. Para isso, utilizaram-se um quadro de madeira de 25 × 25 × $5 \mathrm{~cm}$ de altura e uma pá de jardinagem para auxiliar na remoção do solo, totalizando um volume de $12.500 \mathrm{~cm}^{3}$ por parcela. O material coletado foi armazenado em sacos plásticos pretos, devidamente etiquetados. Galhos e folhas recém-caídos foram excluídos da amostragem, sendo deixada apenas a serapilheira já em estágio inicial de decomposição. As 20 amostras foram levadas ao viveiro do Departamento de Engenharia Florestal da Universidade Federal de Viçosa e subdivididas em 40 caixas de madeira perfuradas na base, de 50 x 50 x $10 \mathrm{~cm}$ de profundidade. As amostras foram divididas de forma que metade delas (20 caixas) fosse disposta em mesas inteiramente recobertas com tela de náilon transparente, proporcionando sombreamento de $11,5 \%$ (luz), e a outra foi mantida em casa de vegetação, recoberta por náilon preto, com sombreamento de $60 \%$ (sombra). Foram distribuídos dois recipientes com areia esterilizada sobre as mesas nos dois ambientes, a fim de monitorar a chegada de sementes, e todas as caixas receberam regas diárias.

\subsection{Análise dos dados}

A análise do banco de sementes foi realizada utilizando-se o método de germinação de Brown (1982). Fez-se a quantificação das plântulas mediante a contagem semanal cumulativa de cada morfoespécie, considerando as formas de vida herbácea, arbustiva, escandente e arbórea. O reconhecimento e identificação das espécies 
foram realizados no viveiro por meio de comparação com as coleções do Herbário VIC/UFV. Adotou-se o sistema de classificação de Cronquist (1981), com exceção das famílias Caesalpiniaceae, Fabaceae e Mimosaceae, que permaneceram como subfamílias de Leguminosae. Os nomes científicos e respectivos autores foram atualizados por meio do site www.mobot.org (Missouri Botanical Garden). De cada morfoespécie, um a cinco indivíduos foram transplantados para saquinhos de repicagem com substrato-padrão para viveiro e cultivados em ambiente de sombra para posterior identificação. Ao longo do experimento foram feitas duas adubações químicas em todo o material de análise. O restante das plântulas de cada morfoespécie foi retirado periodicamente das caixas, a fim de facilitar a contagem.

A emergência das plântulas foi acompanhada durante os meses de abril a outubro de 2004, com o revolvimento do solo ao final do primeiro trimestre. Foi calculado o número de indivíduos, bem como a média de germinação e os parâmetros de densidade e frequência para cada espécie ou morfoespécie. A germinação total das espécies arbustivo-arbóreas foi comparada nas duas condições de sombra (11,5 e 60,0\%), utilizando-se o teste $t$ para amostras independentes (SNEDECOR; COCHRAN, 1967). As espécies arbustivo-arbóreas identificadas foram classificadas em quatro categorias sucessionais, de acordo com Gandolfi et al. (1995) e considerando as propostas de categorização de Santos et al. (1996), Bernacci e Leitão Filho (1996), Martins e Rodrigues (2002) e Martins et al. (2004). Foi incluída, também, a categoria das herbáceas invasoras para as ervas cujo comportamento de chegada no ambiente se refletiu em rápida ocupação e disseminação (LÓPEZ-QUILES; VÁZQUEZ-YANES, 1976). Para cada espécie foi considerada, ainda, a sua síndrome de dispersão, se zoocórica, anemocórica, autocórica ou barocórica, conforme Van Der Pijl (1982) e Barroso et al. (1999).

\section{RESULTADOS E DISCUSSÃO}

\subsection{Composição florística}

Foram reconhecidas 66 espécies, pertencentes a 32 gêneros e 21 famílias. As famílias de maior riqueza foram Asteraceae, com 15 espécies, Melastomataceae com 6 e Solanaceae com 4 (Tabela 1). A riqueza florística aqui encontrada está dentro do limite de 8 a 67 espécies apresentado por Garwood (1989), cujo estudo resultou de uma revisão de trabalhos realizados em florestas tropicais perturbadas.
Considerando apenas as espécies arbustivoarbóreas, Baider et al. (1999) encontraram também alta representatividade da família Melastomataceae, com 10 espécies, compreendendo $52,6 \%$ do total.

As espécies herbáceas foram predominantes, seguidas das arbóreas, arbustivas e lianas (Figura 2a). Entre as morfoespécies herbáceas presentes no banco estavam as pertencentes às famílias Asteraceae, Poaceae e Solanaceae. As asteráceas contribuíram com 36,1\% das espécies invasoras, demonstrando, assim, sua forte influência na contaminação de florestas a partir de sementes dispersas de áreas antropizadas vizinhas.

Quatro grupos ecológicos estiveram presentes na composição do banco de sementes desse trecho da Mata da Praça de Esportes, a saber: ervas e cipós invasores, árvores e arbustos pioneiros, árvores secundárias iniciais e secundárias tardias. Em número de espécies, as invasoras dominaram a flora do banco, seguidas das pioneiras, secundárias iniciais e secundárias tardias (Figura 2b). Entre as espécies arbustivo-arbóreas, a categoria das pioneiras foi predominante (42\%), tanto em número de espécies quanto de indivíduos (Tabela 1), e consequentemente houve também predomínio de sementes zoocóricas (Figura 2c), merecendo destaque as espécies de Melastomataceae, Cecropia hololeuca e Potomorphe sp.

A presença das espécies herbáceas invasoras na área pode ser consequência do histórico de uso agrícola na região, como também da sua fragmentação, o que a tornou mais vulnerável à perturbação antrópica no seu entorno. Além disso, a abertura de clareiras causadas pela queda de árvores mortas, presas a outras pelos cipós, pode ter causado a queda de outras árvores, abrindo espaços maiores dentro da floresta. Esse tipo de distúrbio pode ter dado lugar à ocupação de espécies invasoras, que competiram com a regeneração natural nativa, mas pode ter permitido o estabelecimento de pioneiras e, dessa forma, contribuído com maior riqueza de espécies.

\subsection{Germinação, densidade e frequência}

Das 5.194 plântulas observadas, 3.013 estavam no ambiente de sombra $(\mathrm{P}=0,003)$, destacando Cecropia hololeuca $(\mathrm{P}=0,02)$, Leandra purpurarences $(\mathrm{P}<0,001)$, Miconia $\mathrm{sp} 2(\mathrm{P}=0,006)$, Pterocaulon virgatum $(\mathrm{P}=0,02)$ e Leandra $\mathrm{sp} 2(\mathrm{P}=0,01)$ (Tabela 2$)$. 
Tabela 1 - Relação das espécies arbustivo-arbóreas amostradas no banco de sementes de um trecho da Mata da Praça de Esportes, Viçosa, MG, em ordem alfabética de famílias, gêneros e espécies e respectivos hábitos, categorias sucessionais, síndromes de dispersão e número de indivíduos. FV = Forma de Vida; $\mathrm{Av}=$ Árvore; $\mathrm{Ab}=$ Arbusto; $\mathrm{CS}=$ Categoria Sucessional; $\mathrm{P}$ = Pioneira; I = Invasora; $\mathrm{SC}=$ Sem Caracterização; $\mathrm{Si}=$ Secundária inicial; $\mathrm{St}$ = Secundária tardia; $\mathrm{SD}=$ Síndrome de Dispersão; $\mathrm{An}=$ Anemocórica; $\mathrm{Z}=$ Zoocórica; At = Autocórica; e NI = Número de Indivíduos Total.

Table 1 -Relationship of the arbustive-arboreous species sampled in the seed bank of the Mata da Praça dos Esportes zone, Viçosa, MG, in alfabetic order of the families, genus and species, and their respective habits, sucessional categories and dispersal mode. FV = Life Form; Av = Tree; Ab=Shrub; CS = Successional Categories; $P=$ Pioneer; $I=$ Weed; $S C=$ Without Caracterization; $S i=$ Initial Secondary; $S t=$ Late Secondary; $S D=$ Dispersal Mode; $A n=$ Anemochorous $; Z=$ Zoochorous $;$ At $=$ Autochorous $;$ and NIT = Total Number of Individuals.

\begin{tabular}{|c|c|c|c|c|c|}
\hline Família & Espécie & $\mathrm{FV}$ & $\mathrm{CS}$ & $\mathrm{SD}$ & NI \\
\hline Asteraceae & Asteraceae sp 1 & Av & $\mathrm{P}$ & An & 92 \\
\hline Asteraceae & Asteraceae sp 3 & $\mathrm{Ab}$ & I & An & 14 \\
\hline Asteraceae & Asteraceae sp 4 & $\mathrm{Ab}$ & I & An & 3 \\
\hline Asteraceae & Asteraceae sp 5 & $\mathrm{Ab}$ & I & An & 1 \\
\hline Asteraceae & Bacharis sp & $\mathrm{Ab}$ & I & An & 74 \\
\hline Asteraceae & Pterocaulon virgatum (L.) DC. & $\mathrm{Ab}$ & I & An & 271 \\
\hline Bignoniaceae & Jacaranda sp & Av & $\mathrm{SC}$ & An & 5 \\
\hline Cecropiaceae & Cecropia hololeuca Miq. & Av & $\mathrm{P}$ & Z & 1353 \\
\hline Clusiaceae & Vismia guianensis (Aubl.) Pers. & Av & $\mathrm{P}$ & Z & 1 \\
\hline Euphorbiaceae & Croton urucurana Baill & Av & $\mathrm{P}$ & At & 22 \\
\hline Flacourtiaceae & Casearia $\mathrm{sp}$ & Av & $\mathrm{Si}$ & $\mathrm{Z}$ & 3 \\
\hline Melastomataceae & Leandra purpurarences & $\mathrm{Ab}$ & $\mathrm{P}$ & $\mathrm{Z}$ & 701 \\
\hline Melastomataceae & Leandra sp 1 & $\mathrm{Ab}$ & $\mathrm{P}$ & Z & 95 \\
\hline Melastomataceae & Melastomataceae sp 1 & $\mathrm{Ab}$ & $\mathrm{P}$ & Z & 6 \\
\hline Melastomataceae & Miconia cinnamomifolia (DC.) Naudin & Av & $\mathrm{P}$ & $\mathrm{Z}$ & 461 \\
\hline Melastomataceae & Miconia sp 1 & Av & $\mathrm{P}$ & $\mathrm{Z}$ & 320 \\
\hline Melastomataceae & Tibouchina granulosa (Desr.) Cogn. & Av & $\mathrm{P}$ & $\mathrm{Z}$ & 22 \\
\hline Monimiaceae & Siparuna guianensis Aubl. & Av & St & Z & 3 \\
\hline Moraceae & Chlorophora tinctoria (L.) Gaud. & Av & $\mathrm{SC}$ & $\mathrm{Z}$ & 39 \\
\hline Myrtaceae & Myrtaceae sp 1 & Av & $\mathrm{SC}$ & $\mathrm{Z}$ & 1 \\
\hline Piperaceae & Pothomorphe sp1 & Av & $\mathrm{P}$ & $\mathrm{Z}$ & 245 \\
\hline Rubiaceae & Psychotria sessilis (Vell.) Müll. Arg. & Av & St & $\mathrm{Z}$ & 12 \\
\hline Rutaceae & Dictyoloma vandellianum A. Juss. & Av & $\mathrm{Si}$ & $\mathrm{Z}$ & 3 \\
\hline Rutaceae & Zanthoxylum riedelianum Engl. & Av & $\mathrm{Si}$ & Z & 25 \\
\hline Solanaceae & Solanaceae sp 1 & $\mathrm{Ab}$ & $\mathrm{SC}$ & $\mathrm{Z}$ & 2 \\
\hline Solanaceae & Solanum sp 1 & Av & $\mathrm{P}$ & $\mathrm{Z}$ & 44 \\
\hline Solanaceae & Solanum sp 2 & $\mathrm{Ab}$ & $\mathrm{P}$ & $\mathrm{Z}$ & 1 \\
\hline Tiliaceae & Luehea grandiflora Mart. & Av & $\mathrm{Si}$ & An & 70 \\
\hline Verbenaceae & Aegiphila sellowiana Cham. & Av & $\mathrm{P}$ & $\mathrm{Z}$ & 4 \\
\hline TOTAL & & - & - & - & 3893 \\
\hline
\end{tabular}

O maior número de plântulas observadas no ambiente de sombra pode ter sido consequência das condições mais favoráveis oferecidas por esse tratamento, ou seja, nível de luz suficiente para a germinação dessas espécies e ambiente mais úmido, que impediu o ressecamento do solo. Já aquelas espécies que se desenvolveram melhor no ambiente de luz provavelmente requereram níveis maiores de luz e de temperatura para germinação.

Foram observadas, ainda, diferenças na velocidade de emergência das plântulas de várias espécies, provavelmente associadas a diversos tipos 
Tabela 2 - Parâmetros quantitativos das principais espécies arbustivo-arbóreas amostradas no banco de sementes de um trecho da Mata da Praça de Esportes, Viçosa, MG. DT = Densidade total; I = Indivíduos germinados; S = sob $60 \%$ de sombreamento (Sombra); $\mathrm{L}=$ sob 11,5\% de sombreamento (Luz); MT = Média total de germinação; F1 = Frequência no ambiente de luz; e F2 = Frequência no ambiente de sombra.

Table 2 -Quantitative parameters of the main arbustive-arboreous species sampled in the seed bank of Mata da Praça dos Esportes zone, Viçosa, MG. DT = Total density; $I=$ Germinated Individuals; $S=$ under $60 \%$ of shading (Shade); $L=$ under 11,5\% of shading (Light); $M T=$ Total average; $F 1=$ Frequency in bright enviroment; and $F 2=$ Frequency in shady environment.

\begin{tabular}{|c|c|c|c|c|c|c|}
\hline Espécie & DT $\left(\mathrm{I} / \mathrm{m}^{2}\right)$ & IS & IL & MT & F1 (\%) & F2 (\%) \\
\hline Cecropia hololeuca & 270,6 & 565 & 788 & 34 & 100 & 100 \\
\hline Leandra purpurarences & 140,2 & 170 & 531 & 18 & 100 & 100 \\
\hline Miconia cinnamomifolia & 92,2 & 211 & 250 & 12 & 100 & 100 \\
\hline Miconia sp 2 & 64 & 83 & 237 & 8,1 & 95 & 100 \\
\hline Pterocaulon virgatum & 54,2 & 84 & 187 & 6,8 & 80 & 95 \\
\hline Potomorphe sp & 49 & 111 & 134 & 6,3 & 100 & 100 \\
\hline Leandra sp 2 & 19 & 33 & 62 & 2,4 & 75 & 85 \\
\hline Asteraceae sp 1 & 18,4 & 51 & 41 & 2,3 & 70 & 65 \\
\hline Bacharis sp & 14,8 & 43 & 31 & 1,9 & 90 & 70 \\
\hline Luehea grandiflora & 14 & 57 & 13 & 1,8 & 80 & 30 \\
\hline TOTAL & 736,0 & 1.048 & 2.274 & 93 & 890 & 845 \\
\hline
\end{tabular}

de dormência, como primária e induzida. Entre as principais espécies do banco, C. hololeuca, Poaceae spp e M. cinnamomifolia germinaram em grande quantidade no primeiro trimestre de acompanhamento, diminuindo drasticamente a taxa de germinação ao final do período.

Espécies como Leandra purpurarences, Leandra sp (2), Miconia sp (2), Pterocaulon virgatum e Potomorphe sp tiveram respostas diferentes, emergindo em grande quantidade no segundo trimestre do experimento. Tal comportamento de germinação pode estar associado a uma dormência morfológica, na qual o embrião ainda não está desenvolvido; e a uma dormência morfofisiológica, cujo embrião não se desenvolveu e, ainda, passa por inibição fisiológica de germinação ou, simplesmente, podendo ter sido influenciado pelas baixas temperaturas, já que o primeiro trimestre de acompanhamento correspondeu à estação mais fria do ano em Viçosa.

Outros estudos também demonstraram diferenças na velocidade de germinação entre as espécies do banco. Araújo et al. (2001) observaram maior proporção de sementes germinadas no primeiro mês de acompanhamento; o pico de germinação ocorrendo logo após a exposição à luz; e maior amplitude térmica sugere elevado número de sementes com dormência fisiológica, o que ocorre quando o embrião está completamente desenvolvido, porém não recebe água, temperatura e luz adequadas para iniciar a germinação (BASKIN; BASKIN, 1989). Esse tipo de dormência pode estar relacionado também à presença de serapilheira, conforme observado por Metcalfe e Turner (1998), que relataram que algumas espécies do banco necessitavam apenas de uma ruptura da camada de serapilheira, enquanto outras precisavam de algum distúrbio no solo, simultaneamente à abertura do dossel.

A densidade total observada em uma área amostral de $5 \mathrm{~m}^{2}$ foi de $1.038,8$ sementes $/ \mathrm{m}^{2}$. De acordo com Garwood (1989), a densidade do banco de florestas tropicais secundárias antigas varia de 25 a 3.350 sementes $/ \mathrm{m}^{2}$. Estudando uma floresta madura circundada por florestas em regeneração, Baider et al. (1999) encontraram um valor de 872 sementes $/ \mathrm{m}^{2}$, enquanto Putz e Appanah (1987) detectaram apenas 131 sementes $/ \mathrm{m}^{2}$ em uma floresta madura da Malásia.

Quando se comparam sítios de diferentes idades e histórias de vida, percebe-se claramente essa variação, como foi observado por Baider et al. (2001), que registraram densidades de 11.028, 4.644 e 5.100 sementes $/ \mathrm{m}^{2}$ em sítios de 8,18 e 27 anos de idade, respectivamente. Araújo et al. (2001) encontraram densidades muito menores, de $2.848,1.428$ e 756 sementes $/ \mathrm{m}^{2}$, estudando sítios florestais de 6,17 e 30 anos, respectivamente, o que revela uma diminuição da quantidade de sementes com

Revista Árvore, Viçosa-MG, v.36, n.3, p.423-432, 2012 
o avanço da sucessão. Do mesmo modo, Leal-Filho (1992) observou, em um fragmento florestal em Viçosa, Minas Gerais, que o número de sementes aumentava no sentido Floresta Secundária Madura, capoeira e pasto.

Essas variações na densidade de sementes em áreas diferentes podem estar ligadas a diversos fatores, como o histórico de perturbação, a fonte de propágulos e a qualidade e quantidade da fauna dispersora.

As famílias que se destacaram quanto à abundância foram Melastomataceae, Cecropiaceae, Asteraceae, Poaceae e Piperaceae (1.605, 1.353, 709, 543 e 248 indivíduos, respectivamente). As principais famílias de espécies invasoras foram Asteraceae e Poaceae, que contribuíram, juntas, com $72,7 \%$ das sementes dessa categoria

A alta representatividade da família Melastomataceae, principalmente dos gêneros Miconia, Leandra e Tibouchina, também tem sido observada por outros autores (LEAL-FILHO, 1992; BAIDER et al., 1999; DALLING; DENSLOW, 1998; ARAÚJO et al., 2001; BAIDER et al., 2001; SOUZA, 2003), como consequência do caráter pioneiro da maioria de suas espécies e sua abundância nas florestas semideciduais. M. cinnamomifolia também esteve entre as espécies mais abundantes na amostragem da vegetação arbórea em um fragmento florestal próximo à Mata da Praça de Esportes, no Campus da UFV, demonstrando, assim, sua importância na regeneração dessas florestas (SOUZA, 2003).

Sementes do gênero Cecropia têm sido encontradas em vários trabalhos com banco de sementes (FLEMING; HELTHAUS, 1981; HOLTHUIJZEN; BOERBOOM, 1982; DANIEL; JANKAUSKIS, 1989; LEAL-FILHO, 1992; GROMBONE-GUARATINI, 1994;DALLING;DENSLOW, 1998; DALLING et al., 1998; MEDLÍN; GAONA, 1999; ARAÚJO et al., 2001; SOUZA 2003) e comumente são dotadas de grande longevidade (HOLTHUIZEN; BOERBOOM 1982), refletindo sua importância no processo de regeneração dessas florestas.

As espécies mais frequentes no banco foram representadas, em sua maioria, por pioneiras arbustivoarbóreas, com exceção de Pterocaulon virgatum e Bacharis sp, consideradas invasoras (Tabela 2). Assim, a categoria das pioneiras foi a mais abundante, representando $56,1 \%$ do total de plântulas germinadas, $64,8 \%$ das espécies arbustivo-arbóreas, seguidas das invasoras com 30,5\%, das secundárias iniciais com $1,94 \%$ e das secundárias tardias com $0,3 \% ; 2,3 \%$ permaneceram sem caracterização (Figura 2a).

A maioria dos indivíduos amostrados no banco foi de espécies arbóreas, predominando entre esses as pioneiras, seguidas das arbustivas e herbáceas e em número extremamente inferior ao das lianas, apesar do número representativo de espécies (Figura 2b).

O domínio de espécies invasoras também foi encontrado por Baider et al. (2001), que observaram forte influência das famílias Asteraceae e Poaceae, representando $60,3 \%$ das sementes dessa categoria. O perigo da ocupação por essas espécies após distúrbios é evidente, considerando-se a sua grande capacidade de crescimento, reprodução e disseminação, o que pode dificultar ou, até mesmo, impedir o estabelecimento de nativas menos agressivas, importantes na cicatrização da floresta.

O predomínio de sementes de espécies arbustivoarbóreas pioneiras no banco é comumente relatado na literatura (BAIDER et al., 1999; ENRIGHT, 1985; LAWTON; PUTZ, 1988; HOPKINS et al., 1990).

Os indivíduos de espécies zoocóricas foram maioria nesta pesquisa (Figura 2c). Estudando a dispersão por aves e morcegos em uma floresta e em um campo agricultável no México, Medlín e Gaona (1999) observaram que $50 \%$ das espécies por eles dispersas eram de pioneiras e que as sementes do gênero Cecropia foram as mais numerosas, atingindo $87 \%$ daquelas disseminadas por morcegos e $83 \%$ das propagadas por aves.

A forte dominância de sementes dispersas por animais no banco do trecho estudado pode representar fator de extrema importância no que se refere à manutenção da fauna dispersora de sementes, durante o início da regeneração da floresta após distúrbios. Isso indica ainda que a área de estudo possa estar sendo utilizada como importante oferta de recursos e abrigo para a fauna.

Apesar da presença de espécies invasoras no banco de sementes, a alta densidade de indivíduos de espécies arbóreas e arbustivas pioneiras, como Cecropia hololeuca, as espécies de Melastomataceae, Asteraceae e Piperaceae, importantes para a regeneração da floresta, refletem uma capacidade de resiliência da área estudada diante da ocorrência de distúrbios.

Revista Árvore, Viçosa-MG, v.36, n.3, p.423-432, 2012 


\section{AGRADECIMENTOS}

À Fundação de Amparo à Pesquisa de Minas Gerais (Fapemig), pela bolsa de mestrado de B.K.S. Franco; e ao Conselho Nacional de Desenvolvimento Científico e Tecnológico (CNPq), pela bolsa de produtividade em pesquisa de S.V. Martins.

\section{REFERÊNCIAS}

ARAÚJO, M. M. et al. Densidade e composição florística do banco de sementes do solo de florestas sucessionais na região do Baixo Rio Guamá, Amazônia Oriental. Scientia Florestalis, v.59, p.115-130, 2001.

BAIDER, C.; TABARELLI, M.; MANTOVANI, W. O banco de sementes de um trecho de uma Floresta Atlântica Montana (São Paulo - Brasil). Revista Brasileira de Biologia, v.59, n.2, p.319-328, 1999.

BAIDER, C.; TABARELLI, M.; MANTOVANI, W. The soil seed bank during Atlantic Forest regeneration in Southeast Brazil. Revista Brasileira de Biologia, v.61, n.1, p.35-44, 2001.

BARRoso, G. M. et al. Frutos e sementes: morfologia aplicada à sistemática de dicotiledôneas. Viçosa, MG: Universidade Federal de Viçosa, 1999. 443p.

BASKIN, J. M.; BASKIN, C. C. Physiology of dormancy and germination in relation to seed bank ecology. In: LECK, M. A.; PARKER, V. T.; SIMPSON, R. L. (Org.). Ecology of soil seed banks. San Diego: Academic Press, 1989. p.53-63.

BERNACCI, L. C.; LEITÃO FILHO, H. F. Flora fanerogâmica da floresta da Fazenda São Vicente, Campinas, SP. Revista Brasileira de Botânica, v.19, n.1, p.149-164, 1996.

BROWN, D. Estimating the composition of a forest seed bank: a comparison of the seed extraction and seedling emergence methods. Canadian Journal of Botany, v.70, n.8, p.1603-1612, 1982.

Revista Árvore, Viçosa-MG, v.36, n.3, p.423-432, 2012
BROWN, S.; LUGO, A. E. Tropical secondary forests. Journal of Tropical Ecology, v.6, n.1, p.1-32, 1990.

COCHRANE, M.; SCHULZE, M. D. Fire as a recurrent event in tropical forests of the eastern Amazon: effects on forest structure, biomass, and species composition. Biotropica, v.31, n.1, p.2-16, 1999.

CORRÊA, G. F. Modelo de evolução e mineralogia da fração argila de solos do planalto de Viçosa, MG. 1984. $187 \mathrm{f}$. Dissertação (Mestrado em Ciência Florestal) Universidade Federal de Viçosa, Viçosa, MG, 1984.

CRONQUIST, A. The evolution and classification of flowering plants. New York: The New York Botanical Garden, 1981. 555p.

DALLING, J. W.; DENSLOW, J. S. Soil seed bank composition along a Forest chronosequence in seasonally moist tropical forest, Panama.

Journal of Vegetation Science, v.9, n.5, p.669-678, 1998.

DALLING, J. W.; SWAINE, M. D.; GARWOOD, N. C. Dispersal patterns and seed bank dynamics of pioneer trees in Moist Tropical Forest. Ecology, v.79, n.2, p.564-578, 1998.

DANIEL, O.; JANKAUSKIS, J. Avaliação da metodologia para o estoque de sementes do solo em floresta de Terra Firme na Amazônia brasileira. IPEF, v.41/42, p.18-36, 1989.

EMPRESA BRASILEIRA DE PESQUISA AGROPECUÁRIA - EMBRAPA. Sistema brasileiro de classificação de solos. Brasília: Centro Nacional de Pesquisa de Solos, 1999. 412p.

ENRIGHT, N. Evidence of a soil seed bank under rain forest in New Guinea. Australian Journal of Ecology, v.10, n.1, p.67-71, 1985.

FENNER, M. Seed ecology. London: Chapman and Hall, 1985. 151p.

FLEMING, T. H.; HELTHAUS, E. R. Frugivorous bats, seed shadows, and the structure of Tropical Forests. Biotropica, v.13, p.45-53, 1981.

(Suplemento) 
GANDOLFI, S.; LEITÃO FILHO, H. F.; BEZERRA, C. L. F. Levantamento florístico e caráter sucessional das espécies arbustivo-arbóreas de uma floresta semidecídua no município de Guarulhos, SP. Revista Brasileira de Biologia, v.55, n.4, p.753-767, 1995.

GARWOOD, N. C. Tropical soil seed banks: a review. In: LECK, M. A.; PARKER, V. T.; SIMPSON, R. L. (Org.). Ecology of soil seed banks. San Diego: Academic Press. 1989. p.149-209.

GOLFARI, L. Zoneamento ecológico do Estado de Minas Gerais. Belo Horizonte: Centro de Pesquisa Florestal da Região do Cerrado, 1975.65p.

GROMBONE-GUARATINI, M. T. Banco de sementes de uma Floresta Ripária no Rio Mogi-Guaçú, SP. 1994. 124f. Dissertação (Mestrado em Biologia Vegetal) - Universidade Estadual de Campinas, Campinas, 1994.

HARPER, J. L. Population biology of plants. London: Academic Press, 1977. 892p.

HOLTHUIJZEN, M. A.; BOERBOOM, J. H. A. The Cecropia seedbank in the Surinam lowland rain forest. Biotropica, v.14, n.1, p.62-68, 1982.

HOPKINS, M. S.; TRACEY, J. G.; GRAHAM, A. $\mathrm{W}$. The size and composition of soil seed banks in remmant patches of three structural rainforest types in North Queensland, Australia, Melboure. Australian Journal Ecology, v.15, n.1, p.43-50, 1990.

KAUFFMAN, J. B. Survival by sprouting following fire in tropical forest of the Eastern Amazon. Biotropica, v.23, n.3, p.219-224, 1991.

KLEIN, R. M. Ecologia da flora e vegetação do Vale do Itajaí. Sellowia, v.32, p.165-389. 1980.

LAWTON, R. O.; PUTZ, F. E. Natural disturbance gap-phase in a wind-exposed tropical sloud forest. Ecology, v.69, n.3, p.764-777, 1988.

LEAL-FILHO, N. Caracterização do Banco de Sementes de Três Estádios de uma Sucessão Vegetal na Zona da Mata de Minas Gerais. 1992. 116f. Dissertação (Mestrado em Ciência Florestal) - Universidade Federal de Viçosa, Viçosa, MG, 1992.
LÓPEZ-QUILES, M.; VAZQUEZ-YANES, C. Estudio sobre germinación de semillas em condiciones naturales controladas. In: GÓMEZPOMPA, A. et al. (Org.). Regeneración de selvas. México: Editorial Continental, 1976. p.250-262.

MARTINS, S. V.; RIBEIRO, G. A. Initial secondary succession in a forest fragment disturbed by fire in Viçosa-MG, Brasil. In: Forest Fire

Research \& Wildland Fire Safety. Rotterdam: Milpress, 2002. p.1-9.

MARTINS, S. V.; RODRIGUES, R. R. Gap-phase regeneration in semideciduous mesophytic forest, south-eastern Brazil. Plant Ecology, v.163, n.1, p.51-62, 2002.

MARTINS, S. V. et al. Colonization of gaps produced by death of bamboo clumps in a semideciduos mesophytic forest in south-eastern Brazil. Plant Ecology, v.172, n.1, p.121-131, 2004.

MARTINS, S. V. et al. Banco de sementes como indicador de restauração de uma área degradada por mineração de caulim em Brás Pires, MG. Revista Árvore, v.32, n.6, p.1081-1088, 2008.

MARTINS, S. V. Soil seed bank as indicator of forest regeneration potential in canopy gaps of a semideciduous forest in Southeastern Brazil. In: FOURNIER, M. V. (Ed.) Forest regeneration: ecology, management and economics. New York: Nova Science Publishers, p.113-128, 2009.

MEDLÍN, R.A.; GAONA, O. Seed dispersal by bats and birds in Forest and disturbed habitats of Chiapas, México. Biotropica, v.31, n.3, p.478485, 1999.

MEIRA-NETO, J. A. A. et al. Estrutura de uma Floresta Estacional Semidecidual submontana em área diretamente afetada pela usina hidrelétrica de Pilar, Ponte Nova, Zona da Mata de Minas Gerais. Revista Árvore, v.21, n.3, p.338-344, 1997.

METCALFE, D. J.; TURNER, I. M. Soil seed bank from lowland rain Forest in Singapore: canopygap and litter-gap demanders. Journal of Tropical Ecology, v.14, n.1, p.103-108, 1998.

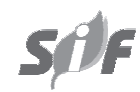

Revista Árvore, Viçosa-MG, v.36, n.3, p.423-432, 2012 
OLIVEIRA FILHO, A. T.; SCOLFORO, J. R.; MELLO, J. M. Composição florística e estrutura comunitária de um remanescente de Floresta Semidecídua Montana em Lavras, MG. Revista Brasileira de Botânica, v.17, n.2, p.167-182, 1994.

PUTZ, F. E.; APPANAH, S. Buried seeds, newly dispersed seeds, and the dynamics of a lowland forest in Malaysia. Biotropica, v.19, p.326-333, 1987.

PUTZ, F. E. Treefall pits and mounds, buried seeds, and the importance of soil disturbance to pioneer trees on Barro Colorado Island, Panama. Ecology, v.64, n.5, p.1069-1074, 1983.

RESENDE, M. Clima e solo: suas relações com o ambiente agrícola. Informe

Agropecuário, v.12, n.138, p.43-49, 1985.

REZENDE, S. B. Estudo de cronotopografia em Viçosa - Minas

Gerais. 1971. 71f. Dissertação (Mestrado em Ciência Florestal) - Universidade Federal de Viçosa, Viçosa, MG, 1971.

SANTOS, F. A. M. et al. The dynamics of tree populations in a semideciduous forest at Santa Genebra reserve, Campinas, SE, Brazil. Supplement to bulletin of the Society of America, v.77, n.3, p.389-341, 1996.

SNEDECOR, G. W.; COCHRAN, W. G. Statistical methods. 6.ed. Ames: lowa College Press, 1967. 593p.
SOUZA, P. A. Efeito da sazonalidade da serapilheira sobre o banco de sementes visando seu uso na Recuperação de Áreas Degradadas. 2003. 130f. Tese

(Doutorado em Ciência Florestal) - Universidade Federal de Viçosa, Viçosa, MG, 2003.

SWAINE, M. D.; HALL, J. B. Early succession on cleared forest land in Ghana. Journal of Ecology, v.71, n.2, p.601-627, 1983.

UHL, C. Factors controlling succession following slash and burn agriculture in Amazonia. Journal of Ecology, v.75, n.2, p.377-407, 1987.

UHL, C. et al. Early plant succession after cutting and burning in the upper Rio Negro Region of the Amazon Basin. Journal of Ecology, v.69, n.2, p.631-649, 1981.

UHL, C.; CLARK, K.; MAQUIRINO, P. Vegetation dynamics in Amazonian treefall gaps. Ecology, v.69, n.3, p.751-763, 1988.

VALVERDE, O. O estudo regional da Zona da Mata de Minas Gerais. Revista Brasileira de Geografia, v.20, n.1, p.1-82, 1959.

van der PIJL, L. Principles of dispersal in higher plants. 3ed. New York: Springer-Verlag, 1982. 214p.

VELOSO, H. P.; RANGEL, A. L. R.; LIMA, J. C. A. Classificação da vegetação brasileira, adaptada a um sistema universal. Rio de Janeiro: IBGE, 1991. 123p.

VIANELLO, R.L. Meteorologia básica e aplicações. Viçosa, MG: Universidade Federal de Viçosa, 1991. 449p. 